\title{
Measurement of the Optical Rotation Angle Using a Rotating-Wave-Plate Stokes Polarimeter
}

\author{
J.-F. Lin ${ }^{1, a}$, Y.-L. Lo ${ }^{2}$, T.-T. Liao ${ }^{3}$, and C.-C. Chang ${ }^{3}$ \\ ${ }^{1}$ Department of Computer Application Engineering, Far-East University, Taiwan \\ ${ }^{2}$ Department of Mechanical Engineering, Cheng-Kung University, Taiwan \\ ${ }^{3}$ Graduate School of Mechanical Engineering, Far-East University, Taiwan
}

\section{Abstract}

A polarimeter based on Stokes-Mueller formalism and rotating-wave-plate Stokes polarimeter is successfully developed to measure the optical rotation angle in a chiral medium. The average relative error in the measured rotation angles of glucose solutions with concentrations ranging from 0 to $1.2 \mathrm{~g} / \mathrm{dl}$ is determined to be $3.78 \%$. The correlation coefficient between the measured rotation angle and the glucose concentration is found to be 0.9995 , while the standard deviation is just 0.00376 degrees. From the sol-gel materials containing $\mathrm{C}_{17} \mathrm{H}_{17} \mathrm{ClO}_{6}$ with concentrations ranging from 0 to $0.0665 \mathrm{~g} / \mathrm{ml}$, the average relative error in the measured rotation angles is determined to be $3.63 \%$. Consequently, the developed system is evaluated with a precision of $5.4 \%$ approximately in rotation angle measurement.

\section{Introductions}

In 1997, Cameron and Cóte [1] designed a glucose sensing digital closed-loop processing system, which is based on the heterodyne interference technique and a lock-in amplifier is used to obtain the rotation angle of the glucose. In 2004, Lin et al. [2] proposed a heterodyne Mach-Zehnder interferometer to enhance the measurement resolution about $6 \times 10^{-5^{\circ}}$. However, Lin's optical configuration is complicated. In 2006, Lo and Yu [3] adopted a liquid-crystal modulator to modulate the azimuth of the linearly polarized light in a sinusoidal signal and developed a new signalprocessing for measuring the glucose concentration. In the proposed optical scheme, a linearly polarized input light of $45^{\circ}$ is incident on two kinds of chiral medium. The Stokes parameters of the output light are detected by a rotating-wave-plate Stokes polarimeter and the algorithm for obtaining rotation angle is derived successfully.

\section{Method}

According to the Stokes-Mueller formalism, the Stokes vector $\hat{S}$ models the polarization state of incident light and the Mueller matrix for a chiral medium is expressed as $M_{C B}$, hence, the Stokes vector $S_{C B}$ of output light is calculated by

\footnotetext{
a e-mail : jacklin@cc.feu.edu.tw
}

This is an Open Access article distributed under the terms of the Creative Commons Attribution-Noncommercial License 3.0, which permits unrestricted use, distribution, and reproduction in any noncommercial medium, provided the original work is properly cited. 
14th International Conference on Experimental Mechanics

$$
S_{C B}=\left[\begin{array}{l}
\mathrm{S}_{0} \\
\mathrm{~S}_{1} \\
\mathrm{~S}_{2} \\
\mathrm{~S}_{3}
\end{array}\right]_{C B}=M_{C B} \hat{S}=\left[\begin{array}{cccc}
1 & 0 & 0 & 0 \\
0 & \cos (2 \gamma) & \sin (2 \gamma) & 0 \\
0 & -\sin (2 \gamma) & \cos (2 \gamma) & 0 \\
0 & 0 & 0 & 1
\end{array}\right]\left[\begin{array}{l}
\hat{S}_{0} \\
\hat{S}_{1} \\
\hat{S}_{2} \\
\hat{S}_{3}
\end{array}\right] .
$$

For input polarization condition is chosen a linear polarization light at $45^{\circ}$ direction, and by applying Cramer's rule, and the optical rotation angle can be determined as

$$
\gamma=\tan ^{-1}\left(S_{1} / S_{2}\right) / 2 .
$$

\section{Experimental results}

In this study, samples containing glucose solutions and sol-gel materials containing griseofulvin $\left(\mathrm{C}_{17} \mathrm{H}_{17} \mathrm{ClO}_{6}\right)$ are prepared. The procedure for fabricating the chiral samples is carefully executed and the calibration of polarimeter is done. The experimental results are shown as below:

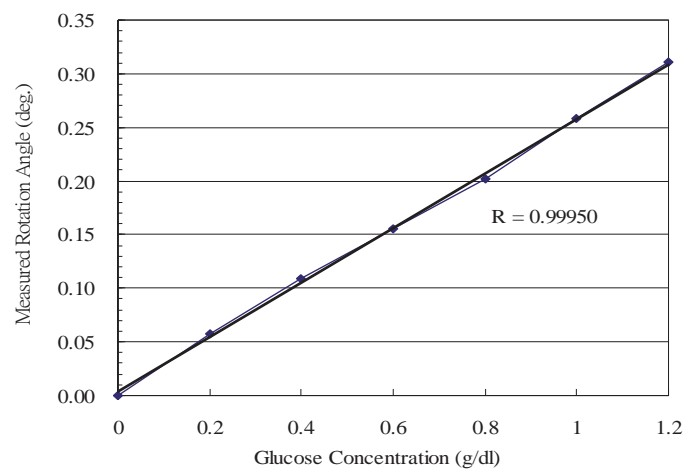

Fig. 1. Variations of rotation angle with glucose concentration (sample: glucose solution).

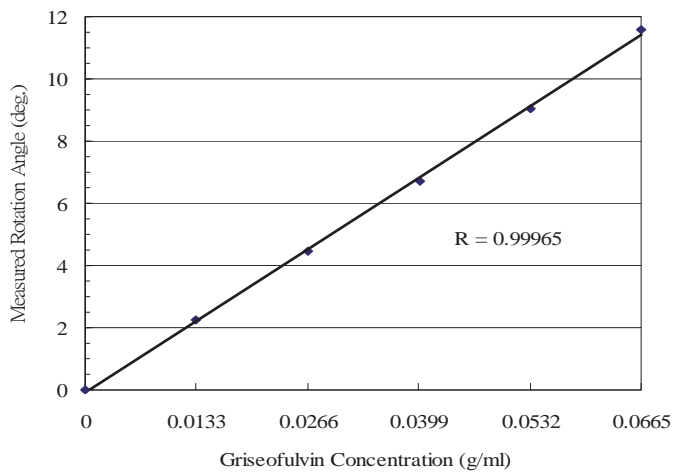

Fig. 2. Variations of rotation angle with chiral griseofulvin concentration (sample: griseofulvin sol-gel).

\section{References}

1. BD Cameron, GL Cóte, IEEE Trans. Biomed. Eng. 44, 1221 (1997)

2. JY Lin, KH Chen, DC Su, Opt. Communi. 238, 113 (2004)

3. YL Lo, TC Yu, Opt. Communi. 259, 40 (2006) 